\title{
Automatic quality assessment for fluorescence microscopy images
}

\author{
Perrine Paul, Dimitrios Kalamatianos, Heiko Duessmann and Heinrich Huber
}

\begin{abstract}
Fluorescence microscopy imaging is a constant trade off between signal to noise ratio, total observation time and spatio-temporal resolution due to photo toxicity. In this paper, we propose a method to estimate the quality of a fluorescent image acquisition, from a single image, taking into account both signal dependent and signal independent noise. We propose a method for the calculation of the signal to noise ratio globally and locally. We validated our algorithm on real experimental data and data with known simulated noise. Results allow us to conclude that this fully automatic method provides a good quantification of the image quality.
\end{abstract}

\section{INTRODUCTION}

Fluorescence Microscopy imaging is a constant trade off between signal to noise ratio (SNR), total observation time and spatio-temporal resolution due to photo toxicity [7]. The purpose of our study is to provide a quantitative way of image quality evaluation, which presents several interests. This value can then be used to give a quantitative feedback to the experimentalist on the quality of the image obtained and in addition for subsequent experiments by determining minimal requirements for automatic segmentation, or by providing a comparison tool among several acquisitions. It can also be used for compression [4] or for feeding a tracking algorithm [14], [15].

Previous studies of SNR in fluorescence microscopy from an image processing point of view can be found in [9], [14], [15]. In all cases, either gaussian or poisson noise alone is consider. In order to differentiate the noise components and to give their respective levels, Bernas and co-workers [4], [5] fitted a quadratic model of the form

$$
V=A+P S+M S^{2}
$$

, where $S$ is the signal corrected for background, $V$ is the estimated noise variance, and $A, P$ and $M$ are the variances of the three noise components considered: additive, Poisson and multiplicative noise, respectively. In [4], the estimation of $A$ was considered inaccurate and moreover bad results were obtained when the background value was 0 . In addition, the parameters fitting was done on times series of 128 images.

In general, noise estimation methods follow two different approaches. The first is the smoothing based approach, where the noise is estimated by the difference between the original

The authors gratefully acknowledge the financial support of the Higher Education Authority.

P. Paul and D. Kalamatianos are with Hamilton Institute, National University of Ireland, Maynooth, Ireland \{perrine.paul dimitris.kalamatianos\}@nuim.ie

H. Duessmann and H. Huber are with the Department of Physiology and Medical Physics, RCSI, York House, York Street, Dublin 2, Ireland \{hduessmann, heinhuber\}@rcsi.ie and the smoothed image, using any adapted noise removal method. The second approach, so called the block-based approach, subdivides the image in blocks, and considers the variance of the most homogeneous blocks in the image as an estimate of the noise. In this study, we use a combination of both methods. We used the block-based method proposed in [3], which is particularly efficient when additive gaussian noise is present. We also used a smoothing based approach, offering a good estimation of the global noise and thus of the signal-dependent noise in the image, using discrete wavelet transform shrinkage to remove the noisy components. A model similar to 1 is then fitted to the estimated noise to compute the level of signal dependant noise. These two approaches were also proposed in [5] as two concurrent estimations of the noise.

In addition, we present a statistical justification of the model presented in [4]. We propose a way to estimate the noise in fluorescence microscope images from a single image, taking into account both signal dependent and signal independent noise. Combining both approaches presented in [5], we present a new expression of the signal to noise ratio (SNR), for both global and local estimation. By estimating the SNR locally, we take into account non uniform repartition of the noise as a result of stronger signal (and thus stronger noise) or possibly some detector faults.

In section II we discuss the different kinds and sources of noise as listed in the literature. A method to automatically estimate the background intensity and the global noise is presented in section III. Section IV describes how we derive a local SNR expression from the global SNR previously calculated. In the section $\mathrm{V}$, we describe the data used to evaluate the performance of our noise estimation method. Finally, our results are summarized in Section VI.

\section{SIGNAL AND NOISE IN FLUORESCENCE MICROSCOPY}

The most common source of noise in fluorescence microscopy is the photon detection noise [9], [5], [4], also known as shot noise or intrinsic noise [16]. Another source of noise is the extrinsic noise. It is composed by the dark current which follows a Poisson distribution, but also by electronics noise and detector-readout noise, both of which follow a Gaussian distribution, and by quantization noise which is characterized by a uniform law [16]. Cross-talk noise may appear by interference from other probes and in order to reduce this noise, in this study, images were acquired using the multi-track method, which generates multi-fluorescence images without crosstalk of emission signals, by means of fast switching between excitations and quasi-simultaneous detection [1] 
To model the noise in fluorescence images, we first need to consider the number of photons $f(u, v)$ detected per pixel $(u, v)$, which can be expressed as [12]

$$
f(u, v)=s(u, v)+\lambda(u, v)
$$

where $s(u, v)$ is the true number of photons sent for the region corresponding to this pixel and $\lambda(u, v)$ denotes the difference between the true and the detected signal, i.e. the noise. According to [12], $f(u, v)$ follows a Poisson distribution of parameter $s(u, v)$, which means that both the mean and the variance of this distribution are $s(u, v)$.

$$
\operatorname{var}(f(u, v))=s(u, v)
$$

The corresponding pixel intensity $I(u, v)$ is the detected number of photons $f(u, v)$ multiplied by the photo-multiplier sensitivity, also called here detector gain $G$. If we consider additional white noise and multiplicative noise, this lead us to the following expression of the image intensity $I$ :

$$
I=G * f+\eta+m * G * s
$$

where $\eta$ is a white additive noise of variance $A$, and $m$ is the multiplicative noise of variance $M$. The global noise $N_{g}$ can then be defined as:

$$
\left.N_{g}(u, v)=I(u, v)-G * s(u, v)\right)
$$

where $G * s(u, v)$ is the noise-free image. Combining Eqs. 24 and 5 we can express the variance of $N_{g}$ as

$$
\operatorname{var}\left(N_{g}\right)=\operatorname{var}(G * \lambda+\eta+m * G * s)
$$

Under the assumption that the additive noise $\eta$, the photonic noise $\lambda$ and the multiplicative noise $m$ are independent, and considering $s(u, v)$, also noted $s$, as deterministic, Eq.6 is equivalent to:

$$
\operatorname{var}\left(N_{g}\right)=G^{2} * \operatorname{var}(\lambda)+\operatorname{var}(\eta)+(G * s)^{2} * \operatorname{var}(m)
$$

As $s(u, v)$ in Eq. 2 is considered to be deterministic, it follows that its variance is null and then combined with Eq. 3 , we can deduct the variance of $\lambda$ :

$$
\operatorname{var}(\lambda(u, v))=\operatorname{var}(f(u, v))=s(u, v)
$$

We can then obtain the expression for the calculation of global noise variance seen in [5], by rewriting Eq.7 as

$$
\operatorname{var}\left(N_{g}(u, v)\right)=G *(G * s(u, v))+A+M *(G * s(u, v))^{2}
$$

where $A$ and $M$ are the variances of additive and multiplicative noises, respectively.

\section{ESTIMATION OF THE GLOBAL SIGNAL TO NOISE RATIO}

The global SNR is estimated in two parts. Firstly, we compute the background noise and mean intensity, based on the estimation of the background variance as proposed in [3]. Secondly, the signal dependent components are computed by wavelet decomposition, using the underlying hypothesis that high frequency corresponds to noise [11], [12], and identifying the level of noise by fitting the model from Eq. 9 .

A. Estimation of the background mean intensity and noise variance

We want to estimate the background noise variance $\sigma_{B g}^{2}$ and the background mean intensity $\bar{I}_{B g}$. For this purpose, we have implemented the algorithm previously proposed in [3]. Firstly, the image is subdivided in blocks of the same size. Each block is analyzed by computing an inhomogeneity measure based on the convolution with 8 masks enhancing 8 directions of edges. The sum of this 8 convolution gives the inhomogeneity measure value for each block. To calculate the estimated background variance $\sigma_{B g}^{2}$ and mean intensity $\bar{I}_{B g}$, an average of the variance and mean is taken in the 20 most homogeneous blocks, i.e. those with the lowest inhomogeneity values. An example of block identified as background with this algorithm is shown in Fig. $1 \mathrm{~b}$

\section{B. Estimation of the global noise $N_{g}$}

The global noise was estimated as the lossy part of the JPEG 2000 compression. We used the codecs implemented in JASPER version 1.900.1 [2]. The image is firstly transformed to the time-frequency domain using Discrete Wavelet Transform with the Daubechies irreversible wavelet waveform [11], [12]. As a consequence of quantization of the wavelet coefficient used for the JPEG 2000, a part of the signal is lost which is assumed to be mainly noise. We then compute the difference between the original image $I$ and the compressed image $I_{j p 2}$ in order to get the estimated global noise per pixel. This global noise is represented as an image of same size as the original image, noted hereby $\tilde{N}_{g}$. Fig. 1c shows a detail of the original image appeared in Fig. 1a, and Fig. 1d shows the same detail after noise removal by wavelet shrinkage. The estimated global noise is shown in Fig. 1e.

\section{Estimation of the Signal Dependent Noise}

We plotted the variance of the estimated global noise against intensity values of the estimated noise-free image and tried to fit the model from Eq. 9. An example of fitting is shown in Fig. 2.

We avoided the false standard deviations due to saturation at maximum level of intensity in a 8 bit image by removing 


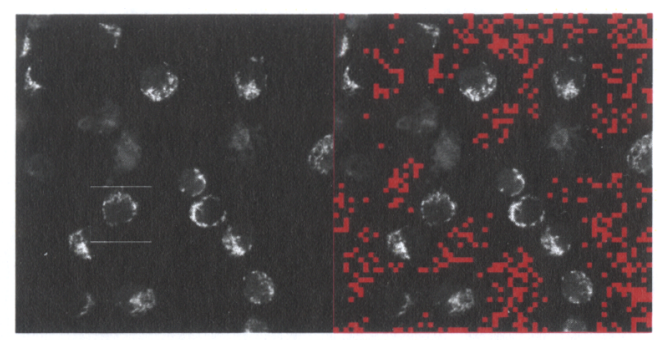

(a)

(b)

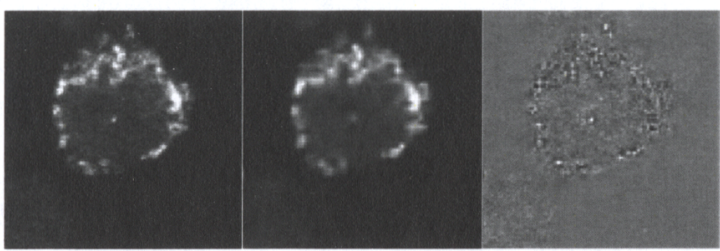

(c)

(d)

(e)

Fig. 1: Different steps of the noise estimation: (a) original image: record 1 of channel with stain GFP, (b) block of pixels identified as background (in red) for estimating $\sigma_{b g}$ and $\bar{I}_{B g}$, (c) detail of original image in the white square, (d) Fig. 1c after wavelet shrinkage with JPEG 2000 compression, (e) estimated noise per pixel as the difference between Figs. 1c and 1d. It is interesting to underline that both released (homogeneous signal) and non released (punctuate signal) CyC-egfp signal were preserved by the JPEG 2000 compression.

this value. In order to estimate the parameters $G$ and $M$, we took $I_{j p 2}$ as an estimate of $G * s$ (the noise-free image), and we used $\tilde{N}_{g}$ as an estimate of the global noise. We imposed $A$ to get the value of the background noise variance $\sigma_{B g}^{2}$, which means that we neglicted for the fitting the signal-dependant noise in the background. Indeed, the background mean intensity values are very low compared to the signal emitted by cells. We then solved numerically the following constrained linear least-square problem, using the preconditioned conjugate gradient optimization algorithm [6], to estimate $G$ the level of Poisson noise (gain estimator) and the variance of the multiplicative noise $M$ :

$$
\begin{gathered}
\min _{G, A} \frac{1}{2}\left\|\overrightarrow{\sigma_{g}^{2}}-\left(\sigma_{B g}^{2}+G \vec{I}+M \vec{I}^{2}\right)\right\|_{2}^{2} \\
\text { such that } G \geq 0, M \geq 0 .
\end{gathered}
$$

and with initial conditions $G=1, M=0$,

where

- $\|.\|_{2}$ is the $L_{2}$-norm

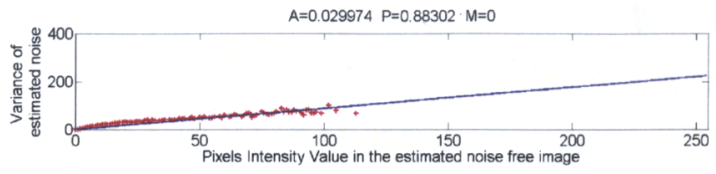

(a)

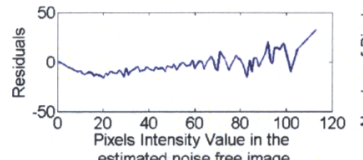

(b)

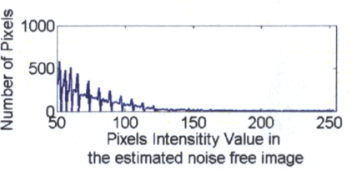

(c)
Fig. 2: Example of Model fitting on one image: (a) Fitting of the model from Eq. 9 to the variance of noise $\tilde{N}_{g}$ for each value of the signal $I_{j p 2}$ (the noise free image) noted as red crosses. (b) Residuals of the model fitting. (c) Number of pixels per intensity value in $I_{j p 2}$. When there were less than 84 pixels, the intensity value was not taken into account for the fitting.

- $\vec{I}$ is a vector of intensity from 0 to 254 , in which values with more than 84 pixels were removed. This threshold was given in [10] as the minimum sample size to obtain an estimated standard deviation into $10 \%$ of its true value with a confidence in the Chi-square sense of $80 \%$.

- The vector $\overrightarrow{\sigma_{g}}$ was constructed as follows: for each value $i$ from 0 to 254 , we computed the variance of the subset of noise values $\tilde{N}_{g}(p(i))$, where $p(i)$ was the subset of pixels whose intensity values in $I_{j p 2}$ were equal to $i$.

\section{Expression of the signal to noise ratio}

The expression of the SNR in decibel was chosen to be as follows:

$$
\mathrm{SNR}_{G}=20 \log _{10}\left(\frac{\bar{I}_{j p 2}-\bar{I}_{B g}}{\sqrt{\operatorname{var}\left(N_{g}\right)}}\right)
$$

where $\bar{I}_{j p 2}$ is the mean value of $I_{j p 2}$. As we have computed both the signal dependent and background noise in sections III-A and III-C, and assumed that background noise was uniformly distributed in the image, we can replace $\operatorname{var}\left(N_{g}\right)$ in Eq. 11 by its value from Eq. 9:

$$
\mathrm{SNR}_{G}=20 \log _{10}\left(\frac{\bar{I}_{j p 2}-\bar{I}_{B g}}{\sqrt{\sigma_{B g}^{2}+G * \bar{I}_{j p 2}+M * \bar{I}_{j p 2}^{2}}}\right)
$$

\section{ESTIMATION OF THE LOCAL SIGNAL TO NOISE RATIO}

To obtain local image quality information, we first divided the image in smaller squares and then we computed a local SNR per sub-region. The size of the block can be 
interactively changed by the user if needed. The expression of the local SNR for each block $b l$ was derived from Eq.12:

$$
\begin{gathered}
\operatorname{SNR}_{L}(b l)= \\
20 \log _{10}\left(\frac{\bar{I}_{j p 2}(b l)-\bar{I}_{B g}}{\sqrt{\sigma_{B g}^{2}(b l)+G(b l) * \bar{I}_{j p 2}(b l)+M(b l) * \bar{I}_{j p 2}(b l)^{2}}}\right)
\end{gathered}
$$

where $\bar{I}_{j p 2}(b l)$ is the mean intensity in the block on which the local SNR is calculated, and the parameters $\sigma_{B g}^{2}(b l), G(b l)$ and $M(b l)$ are computed per block. If only one image is used, then the value estimated on the whole image is used for each block too.

The quality of the image could be non uniform due to uneven illumination for example. Moreover, the signal for each cell can be of different intensity because of the focus plan, the fluorescence stain and activity of the cell, as well as some faults from the Photo-Multiplier (PMT) detector. In order to detect these faults, we can use several acquisitions keeping the same microscope parameters in order to get sufficient number of points for model fitting to get an estimated noise level per block rather than a global level.

\section{DATA SETS USED FOR PERFORMANCE EVALUATION}

We used two data sets to study the global SNR. The first set contains real noise created by tuning the parameters of the microscope and the second one contains simulated noise added to microscope image. The microscope used for this series of experiments was a Zeiss LSM 510 with the following objective: Plan-Apochromat $63 \mathrm{x} / 1.4$ oil DIC. The cells were HeLa D98 stably expressing CytochromeC-eGFP fusion protein [8]. The staining was Hoechst (100 $\mathrm{nM}, 45 \mathrm{~min}$ ), detected in channel noted $\mathrm{H}$, Green Fluorescent Protein, detected in channel noted GFP, and Mitotracker Red $(50 \mathrm{nM}, 20 \mathrm{~min})$, detected in channel noted MTR [8]. We used 8-bit gray scale images, with a size of $512 \times 512$.

\section{A. DataSet 1: Real Noise}

The first data set consists of 6 records with 3 channels measuring the previously staining: channel $\mathrm{H}$, channel GFP and channel MTR. The 6 records were created augmenting the level of noise by changing the photomultiplier (PMT) sensitivity, also called detector gain, the pixel dwell time, the number of scan per image, and the acousto-optic tunable filter (AOTF) expressed relatively to the initial power for the different wavelength, as described in Table I. The post PMT offset, the post PMT analog gain, and the different filters, pinholes and resolution were not changed for the different acquisitions.
TABLE I: Microscope settings during image tests acquisitions for data set 1

\begin{tabular}{|c||c||c||c||c||c||c|}
\hline Records & 1 & 2 & 3 & 4 & 5 & 6 \\
\hline Detector gain & & & & & & \\
channel MTR & 750 & 750 & 850 & 850 & 950 & 1050 \\
channel GFP & 650 & 650 & 750 & 750 & 850 & 950 \\
channel H & 650 & 650 & 750 & 750 & 850 & 950 \\
\hline Exposure Time & 3.2 & 3.2 & 3.2 & 1.6 & 1.6 & 1.6 \\
\hline Average Scan & 4 & 1 & 1 & 1 & 1 & 1 \\
\hline AOTF (in \%) & & & & & & \\
$543 \mathrm{~nm}$ & 20 & 20 & 10 & 10 & 5 & 3 \\
$488 \mathrm{~nm}$ & 10 & 10 & 5 & 5 & 2 & 1 \\
$405 \mathrm{~nm}$ & 3 & 3 & 1.5 & 1.5 & 0.8 & 0.3 \\
\hline
\end{tabular}

\section{B. Data Set 2: Simulated Noise}

Simulated noise was added to the first image of the real data. For this purpose, the channel $\mathrm{H}$ (staining Hoechst) was used because it was the one with the least amount of noise. In order to test our algorithm's ability to identify noise components, we produced data with different kinds of noise. In particular, Poisson, Gaussian noise, and a combination of both were added to the image. To check the influence of the number of blocks used, the same level of noise was simulated 5 times creating a test image 5 times bigger than the original one. In the real data, the background mean value was close to 0 . However, changing the experiment set up parameters would lead to a background value different than 0 , but still as low as possible in order to obtain maximum contrast. Poisson noise could also then appear in the background. To test our algorithm in this case, we run the same simulation after adding an offset of 5 (for 256 gray levels) to the original image.

\section{RESULTS}

For all performance evaluations, the computation time was less than 2 seconds for a $512 \times 512$ 8-bit image using a 2.33 $\mathrm{GHz}$ processor with $2 \mathrm{~GB}$ of RAM. The variance of the multiplicative noise $M$ was in all cases zero or less than $10^{-6}$, in accordance with the results shown in [5].

\section{A. Performance evaluation on real noise}

The results of our estimation on data set 1 are shown in Fig. 3. The Poisson noise is strongly signal-dependent and its level is expected to be proportional to the gain of the microscope photon detectors. Indeed, this is evident in Figs. 3a,3c, and $3 \mathrm{e}$, meaning that this kind of noise can be identified. As it can be seen in Figs. $3 \mathrm{~d}$ and $3 \mathrm{~b}$, the SNR is also mainly influenced by the detector gain. The pixel dwell time was changed between record 3 and 4 and this had a limited influence on the SNR. The SNR for the channel 
MTR rapidly decreased as seen in Fig. $3 \mathrm{f}$ but these results are coherent with visual evaluation and with the SNR computed using the first record as reference signal by ImageJ [13]: the SNR in $\mathrm{dB}$ is $7.1,1.5,-0.24,-4.0$, and -6.4 for records 2 to 6 respectively when our results for these records are 7.32 , $0.99,-0.10,-2.50$, and -2.94 . We used no reference image to obtain these results unlike the ImageJ plugin.

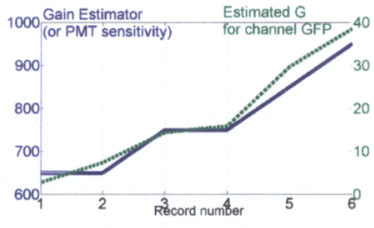

(a)

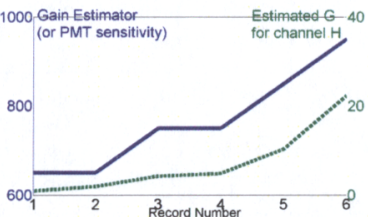

(c)

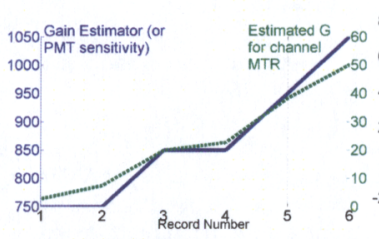

(e)

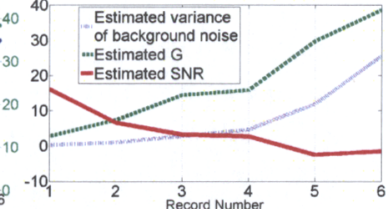

(b)

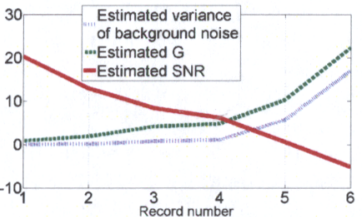

(d)

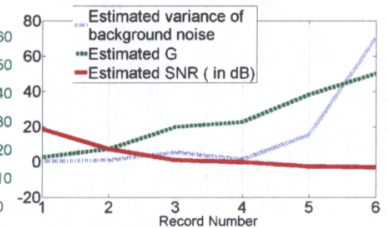

(f)
Fig. 3: Results for data set 1: Poisson noise parameter $G$ against the real detector gain for (a) GFP channel (c) $\mathrm{H}$ Channel (e) MTR Channel; Poisson noise parameter, background noise parameter and global Signal to Noise Ratio for (b) GFP channel (d) H Channel and (f) MTR Channel

\section{B. Performance evaluation on simulated noise}

Results from the estimation of noise on data set 2 are graphically presented in Fig. 4. The relative level of signal dependent noise and background noise was well evaluated. When background was 0 , and only the Gaussian noise level was modified, the estimated Poisson noise was stable and the estimated background noise was varying accordingly. When only Poisson noise was modified, then the estimated background noise was stable and the estimated Poisson noise level was varying accordingly. The presence of a nonzero background influences mainly the estimated background

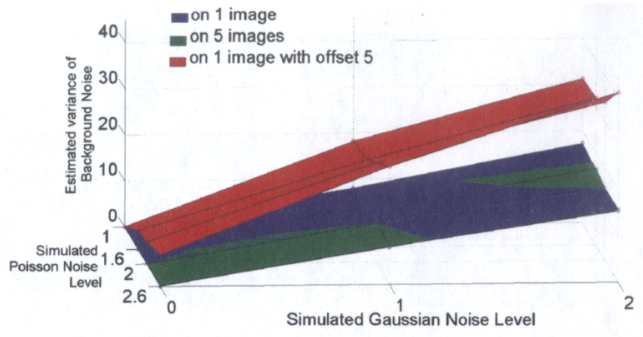

(a)

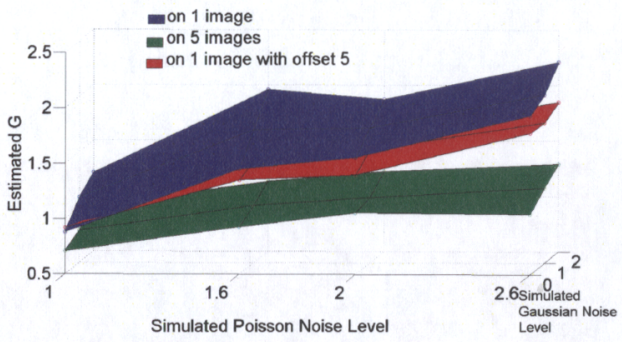

(b)

Fig. 4: Results on data set 2: (a) Estimated variance of the background noise and (b) estimated level of Poisson noise parameter $G$ against the simulated level of both Gaussian and Poisson noise

noise. In this case, in addition to the added Gaussian noise, the background presents a Poisson noise component with a variance proportional to its mean intensity value. The method used to compute the background noise gave the same results when only one or 5 images were used. The estimated Poisson noise is lower when estimated using 5 images, which means that with only one image, we could slightly overestimate the Poisson noise. Thus, the more images available the more robust will be the method, even if useful results are already obtained with a single image.

\section{Visualization of the local signal to noise ratio}

Using our expression 13 enhances the signal level relatively to the noise rather than the noise level as shown in Fig. 5. In order to detect a local failure of the detector, more than one image is needed. Indeed, our algorithm for Poisson noise detection needs each pixel value to contain at least 84 pixels and it is unlikely to be the case in each sub-block of the image. We then simulated a series of 10 images with an added local Poisson noise and applied the algorithm for the global SNR to the concatenation of 10 sub-blocks each time. The corresponding color map of the local SNR is shown on Fig. 6. 


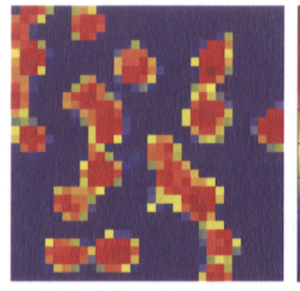

(a)

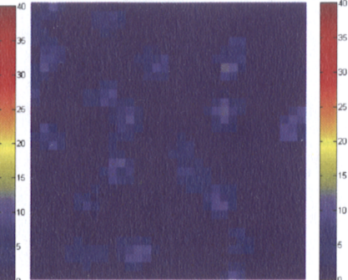

(b)
Fig. 5: Color map of the local SNR in $\mathrm{dB}$ computed from Eq. 13 for a block size of $2^{4}=16$, for (a) channel MTR record 1 and (b) channel MTR record 6.

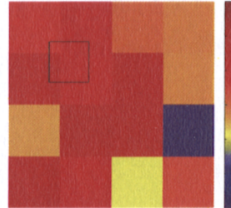

(a)

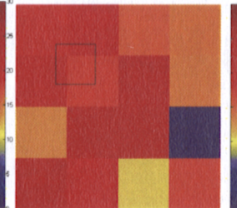

(b)

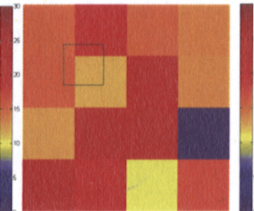

(c)
Fig. 6: Color map of the SNR by block in $\mathrm{dB}$ computed with 10 images for a block size of $2^{7}=128$, for record 1 of GFP channel (a) original (b) with simulated Poisson noise locally in the black rectangle (c) with simulated Gaussian noise locally in the black rectangle

\section{CONCLUSIONS AND FUTURE WORKS}

We have proposed a new method to identify the signal dependent and signal independent noise components from a static image, adapted from [5] and [4]. We have shown that this method gives a good estimation of the SNR and the level of the different noise components. The purpose here is not to evaluate the real detector gain, since we know it from the microscope settings, but to evaluate the resulting noise in the obtained images. Experimental images contain mainly Poisson noise, but there are also additive components that can not be neglected. The robustness of the noise computation can be increased by using additional image by simply adding more points the noise-intensity fitting curve adding pixels from other images taken with the same microscope settings, but with a linear increase on the computational cost. We have also proposed a new method to calculate the SNR of an image locally, and thus allowing the local calibration of the image set up. All methods implemented in this study are fully automatic and the only parameter to be tuned for the convenience of the user is the block size for the estimation of the local SNR. Our method does not remove the noise, but instead smooths the image, comprising the signal dependent noise and the wavelet shrinkage based on entropy offered by JPEG 2000. Due to the limitations of this compression, if the image is too noisy, then the estimated noise-free image will still contain noise and the model fitting will not give correct results. Moreover, the estimated noise-free image can present blurred and then wider edges, which will result in an overestimation of the variance of noise. A possible extension could be to use more efficient noise removal methods. However, despite these limitations, this method could be useful for experimentalists and image analysts working in the field of fluorescence microscopy.

\section{REFERENCES}

[1] LSM 510: Eliminating Crosstalk by Multitracking. ZEISS, micro info 40-520 e/10.99. available from www.zeiss.com.

[2] M.D. Adams. Jasper software reference manual (version 1.900.0). Technical report, ISO/IEC JTC 1/SC 29/WG 1N 2415, 2006.

[3] A. Amer and E. Dubois. Fast and reliable structure-oriented video noise estimation. IEEE Trans. Circuits Syst. Video Technol., 15(1):113-18, 2005.

[4] T. Bernas, E.K. Asem, J.P. Robinson, and B. Rajwa. Compression of fluorescence microscopy images based on the signal-to-noise estimation. Microsc. Res. Tech., 69:1-9, 2006.

[5] T. Bernas, D. Barnes, E.K. Asem, Robinson J.P., and B. Rajwa. Precision of light intensity measurement in biological optical microscopy. Journal of Microscopy, 226:163-74, 2007.

[6] T.F. Coleman and A. Verma. A preconditioned conjugate gradien approach to linear equality constrained minimization. Computational Optimization and Applications, 20(1):61-72, 2001.

[7] J.F. Dorn, G. Danuser, and G. Yang. Methods in Cell Biology, volume 85, chapter Computational Processing and Analysis of Dynamic Fluorescence Image Data, pages 497-538. Elsevier, 2008.

[8] H. Duessmann, M. Rehm, D. Kgel, and J.H.M. Prehn. Outer mitochondrial membrane permeabilization during apoptosis triggers caspase-independent mitochondrial and caspase-dependent plasma membrane potential depolarization: a single-cell analysis. J. Cell Sci. 116(3):525-36, 2003.

[9] Y. Garini, V. Gil, I. Bar-Am, D. Cabib, and N. Katzir. Signal to noise analysis of multiple color fluorescence imaging microscopy. Cytometry, 35:214-26, 1999.

[10] J.A. Greenwood and M.M. Sandomire. Sample size required for estimating the standard deviation as a per cent of its true value. Journal of the American Statistical Association, 45:257-60, 1950.

[11] X. Huang, A.C. Madoc, and A.D. Cheetham. Wavelet-based bayesian estimator for poisson noise removal from images. In IEEE International Conference on Multimedia \& Expo, 2003.

[12] R.D. Nowak and R.G. Baraniuk. Wavelet-domain filtering for photon imaging systems. IEEE Trans. Image Process., 8:666-78, 1999.

[13] D. Sage. SNR: ImageJ plugin to compute the signal-to-noise ratio on images. http://bigwww.epfl.ch/sage/soft/snr/, 29 february 2008 .

[14] D. Sage, F.R. Neumann, F. Hediger, S.M. Gasser, and M. Unser. Automatic tracking of individual fluorescence particles: Application to the study of chromosome dynamics. IEEE Trans. Image Process. 14(0): 1372-83, 2005.

[15] I.F. Sbalzarini and P. Koumoutsakos. Feature point tracking and trajectory analysis for video imaging in cell biology. J. Struct. Biol. 151(2):182-195, 2005

[16] G. van Kempen. Image Restoration in Fluorescence Microscopy. $\mathrm{PhD}$ thesis, Delft University, 1999. 